\title{
An Exact Line Integral Representation of the Magnetic Physical Optics Scattered Field
}

Meincke, Peter; Breinbjerg, Olav; Jørgensen, Erik

Published in:

I E E E Transactions on Antennas and Propagation

Link to article, DOI:

10.1109/TAP.2003.812185

Publication date:

2003

Document Version

Publisher's PDF, also known as Version of record

Link back to DTU Orbit

Citation (APA):

Meincke, P., Breinbjerg, O., \& Jørgensen, E. (2003). An Exact Line Integral Representation of the Magnetic Physical Optics Scattered Field. I E E E Transactions on Antennas and Propagation, 52(6), 1395-1398. https://doi.org/10.1109/TAP.2003.812185

\section{General rights}

Copyright and moral rights for the publications made accessible in the public portal are retained by the authors and/or other copyright owners and it is a condition of accessing publications that users recognise and abide by the legal requirements associated with these rights.

- Users may download and print one copy of any publication from the public portal for the purpose of private study or research.

- You may not further distribute the material or use it for any profit-making activity or commercial gain

- You may freely distribute the URL identifying the publication in the public portal 


\section{Communications}

\section{An Exact Line Integral Representation of the Magnetic Physical Optics Scattered Field}

Peter Meincke, Olav Breinbjerg, and Erik Jørgensen

\begin{abstract}
An exact line integral representation is derived for the magnetic physical optics field scattered by a perfectly electrically conducting planar plate illuminated by electric or magnetic Hertzian dipoles. The positions of source and observation points can be almost arbitrary. Numerical examples are presented to illustrate the exactness of the line integral representation.
\end{abstract}

Index Terms-Line integral, magnetic field, physical optics.

\section{INTRODUCTION}

The transformation of surface integrals representing scattered or diffracted fields into line integrals has achieved much attention in the past decades [1]-[8]. By extending the work by Asvestas [4]-[6], the traditional surface integral of the electric physical optics (PO) scattered field from a perfectly electrically conducting (PEC) planar plate illuminated by an electric Hertzian dipole was cast into a line integral along the edges of the plate in [7]. The procedure of [7] was recently extended in [8] to derive a line integral representation of the electric PO scattered field from a penetrable planar plate illuminated by a plane wave.

In [7] only the electric PO scattered field was considered. However, in some applications it is also necessary to know the magnetic PO scattered field from PEC planar plates. To the knowledge of the authors, a line integral representation of this magnetic field has not been previously published in the literature. In the present letter we derive such a line integral representation and we consider illumination by an electric as well as a magnetic Hertzian dipole. Although the derivation of this letter closely follows that of [7] the form of the final result (12) for the magnetic PO field is significantly different from that of the corresponding expression [7, (24)] for the electric PO field. The letter is a continuation of [7] and several references to this paper are therefore made in the following. Throughout the letter the time factor $\exp (j \omega t)$ is assumed and suppressed.

\section{LINE INTEGRAL REPRESENTATION}

Consider the planar PEC plate $A$ in Fig. 1. A rectangular $x y z$ coordinate system is introduced such that the plate is located in the $x y$ plane and such that $\hat{\mathbf{z}}$ is the normal unit vector. An electric or a magnetic Hertzian dipole with position vector $\mathbf{r}_{S}$ is located in the region $z>0$. The observation point $F$ with position vector $\mathbf{r}_{F}$ can be located everywhere in space, except on the plate. The magnetic PO scattered field $\mathbf{H}^{P O}$ at this observation point is in terms of the traditional PO surface integral given by

$$
\mathbf{H}^{P O}\left(\mathbf{r}_{F}\right)=\nabla \times \int_{A} 2 \hat{\mathbf{z}} \times \mathbf{H}^{i}\left(\mathbf{r}^{\prime}\right) G\left(\mathbf{r}_{F}, \mathbf{r}^{\prime}\right) d A^{\prime}
$$

Manuscript received June 20, 2000; revised May 7, 2002.

The authors are with Ørsted-DTU, Electromagnetic Systems, Technical University of Denmark, DK-2800 Kgs. Lyngby, Denmark (email: pme@oersted.dtu.dk).

Digital Object Identifier 10.1109/TAP.2003.812185

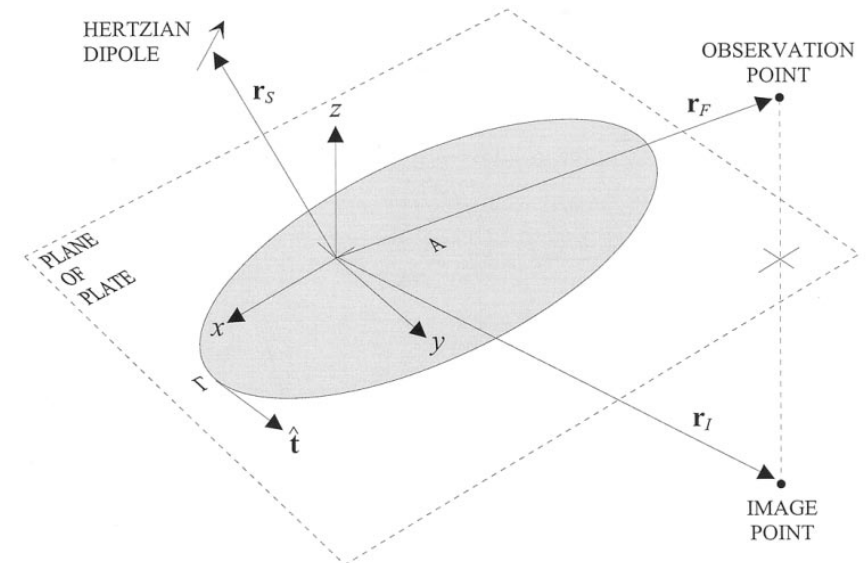

Fig. 1. Planar PEC plate illuminated by an electric or a magnetic Hertzian dipole.

where $\mathbf{r}^{\prime}$ is the position vector of the integration point, $\mathbf{H}^{i}$ is the incident magnetic field, and

$$
G\left(\mathbf{r}, \mathbf{r}^{\prime}\right)=\frac{\exp (-j k R)}{4 \pi R}, \quad R=|\mathbf{R}|, \mathbf{R}=\mathbf{r}^{\prime}-\mathbf{r}
$$

is the scalar Green function for the ambient homogeneous medium with $k$ being the wave number. Introducing the image point $I$, with position vector $\mathbf{r}_{I}$, of the observation point $F$ with respect to the plane of the plate, (1) can be written as [9]

$$
\mathbf{H}^{\mathrm{PO}}\left(\mathbf{r}_{F}\right)=\mathbf{H}^{A}\left(\mathbf{r}_{F}\right)-(\overline{\mathbf{I}}-2 \hat{\mathbf{z}} \hat{\mathbf{z}}) \cdot \mathbf{H}^{A}\left(\mathbf{r}_{I}\right)
$$

where $\overline{\mathbf{I}}$ is the unit dyad

$$
\begin{aligned}
\mathbf{H}^{A}(\mathbf{r})= & \nabla \times \int_{A} \hat{\mathbf{z}} \times \mathbf{H}^{i}\left(\mathbf{r}^{\prime}\right) G\left(\mathbf{r}, \mathbf{r}^{\prime}\right) d A^{\prime} \\
& +\frac{j}{k Z} \nabla \times \nabla \times \int_{A} \hat{\mathbf{z}} \times \mathbf{E}^{i}\left(\mathbf{r}^{\prime}\right) G\left(\mathbf{r}, \mathbf{r}^{\prime}\right) d A^{\prime}
\end{aligned}
$$

and $Z$ is the intrinsic impedance. $\mathbf{H}^{A}$ is the magnetic field from the electric and magnetic surface currents $\hat{\mathbf{z}} \times \mathbf{H}^{i}$ and $-\hat{\mathbf{z}} \times \mathbf{E}^{i}$, respectively, located on the plate $A$. Using the fact that the observation point is assumed not to be on the plate, we can interchange the curl and integration operators in (4) and use the fact that $G$ satisfies the homogeneous wave equation, along with the relation $\nabla G\left(\mathbf{r}, \mathbf{r}^{\prime}\right)=-\nabla^{\prime} G\left(\mathbf{r}, \mathbf{r}^{\prime}\right)=$ $G\left(\mathbf{r}, \mathbf{r}^{\prime}\right)(j k+1 / R) \hat{\mathbf{R}}$ with $\hat{\mathbf{R}}=R^{-1} \mathbf{R}$, to write (4) as

$$
\begin{aligned}
\mathbf{H}^{A}(\mathbf{r})= & \int_{A}\left[\hat{\mathbf{z}} \times \mathbf{H}^{i}\left(\mathbf{r}^{\prime}\right)\right] \times \nabla^{\prime} G\left(\mathbf{r}, \mathbf{r}^{\prime}\right) d A^{\prime}+\frac{j}{k Z} \int_{A}\left[\left[\hat{\mathbf{z}} \times \mathbf{E}^{i}\left(\mathbf{r}^{\prime}\right)\right]\right. \\
& \left.\cdot \nabla^{\prime} \nabla^{\prime} G\left(\mathbf{r}, \mathbf{r}^{\prime}\right)+\hat{\mathbf{z}} \times \mathbf{E}^{i}\left(\mathbf{r}^{\prime}\right) k^{2} G\left(\mathbf{r}, \mathbf{r}^{\prime}\right)\right] d A^{\prime} .
\end{aligned}
$$

We now use the relation

$$
\begin{aligned}
\frac{j}{k Z}\left(\hat{\mathbf{z}} \times \mathbf{E}^{i}\right) \cdot \nabla^{\prime} \nabla^{\prime} G & =\frac{j}{k Z} \hat{\mathbf{z}} \cdot\left(\mathbf{E}^{i} \times \nabla^{\prime} \nabla^{\prime} G\right) \\
& =\frac{1}{j k Z} \hat{\mathbf{z}} \cdot \nabla^{\prime} \times\left(\mathbf{E}^{i} \nabla^{\prime} G\right)+\hat{\mathbf{z}} \cdot \mathbf{H}^{i} \nabla^{\prime} G
\end{aligned}
$$


along with Stoke's dyadic theorem, to rewrite the second integral in (5), into

$$
\begin{aligned}
\mathbf{H}^{A}(\mathbf{r})=\int_{A}[ & {\left[\hat{\mathbf{z}} \times \mathbf{H}^{i}\left(\mathbf{r}^{\prime}\right)\right] \times \nabla^{\prime} G\left(\mathbf{r}, \mathbf{r}^{\prime}\right) } \\
& +\hat{\mathbf{z}} \cdot \mathbf{H}^{i}\left(\mathbf{r}^{\prime}\right) \nabla^{\prime} G\left(\mathbf{r}, \mathbf{r}^{\prime}\right) \\
& \left.+\frac{j k}{Z} \hat{\mathbf{z}} \times \mathbf{E}^{i}\left(\mathbf{r}^{\prime}\right) G\left(\mathbf{r}, \mathbf{r}^{\prime}\right)\right] d A^{\prime} \\
+ & \frac{1}{j k Z} \int_{\Gamma} \hat{\mathbf{t}} \cdot \mathbf{E}^{i}\left(\mathbf{r}^{\prime}\right) \nabla^{\prime} G\left(\mathbf{r}, \mathbf{r}^{\prime}\right) d \Gamma^{\prime}
\end{aligned}
$$

with $\Gamma$ denoting the edge of the plate and $\hat{\mathbf{t}}$ being the edge unit tangent vector related to $\hat{\mathbf{z}}$ via the right-hand rule. This expression is the modified Stratton-Chu representation of the magnetic field. Upon using the fact that the Hertzian dipole is not located on the plate we obtain for the third term in the surface integral of (7)

$$
\begin{aligned}
\frac{j}{k Z} \hat{\mathbf{z}} \times \mathbf{E}^{i} G= & \hat{\mathbf{z}} \times\left[\nabla^{\prime} \times\left(\mathbf{H}^{i} G\right)\right]+\hat{\mathbf{z}} \cdot \nabla^{\prime} G \mathbf{H}^{i}-\hat{\mathbf{z}} \cdot \mathbf{H}^{i} \nabla^{\prime} G \\
= & \left(\hat{\mathbf{z}} \times \nabla^{\prime}\right) \times\left(\mathbf{H}^{i} G\right)-\left(\hat{\mathbf{z}} \times \mathbf{H}^{i}\right) \times \nabla^{\prime} G \\
& -G \hat{\mathbf{z}} \cdot \nabla^{\prime} \mathbf{H}^{i}+\hat{\mathbf{z}} \cdot \nabla^{\prime} G \mathbf{H}^{i}-\hat{\mathbf{z}} \cdot \mathbf{H}^{i} \nabla^{\prime} G .
\end{aligned}
$$

Applying this result along with Stoke's extended theorem [10], (7) becomes

$$
\begin{aligned}
\mathbf{H}^{A}(\mathbf{r})= & -\int_{A} \hat{\mathbf{z}} \cdot\left[G\left(\mathbf{r}, \mathbf{r}^{\prime}\right) \nabla^{\prime} \mathbf{H}^{i}\left(\mathbf{r}^{\prime}\right)-\nabla^{\prime} G\left(\mathbf{r}, \mathbf{r}^{\prime}\right) \mathbf{H}^{i}\left(\mathbf{r}^{\prime}\right)\right] d A^{\prime} \\
& +\int_{\Gamma} \hat{\mathbf{t}} \times \mathbf{H}^{i}\left(\mathbf{r}^{\prime}\right) G\left(\mathbf{r}, \mathbf{r}^{\prime}\right) d \Gamma^{\prime} \\
& +\frac{1}{j k Z} \int_{\Gamma} \hat{\mathbf{t}} \cdot \mathbf{E}^{i}\left(\mathbf{r}^{\prime}\right) \nabla^{\prime} G\left(\mathbf{r}, \mathbf{r}^{\prime}\right) d \Gamma^{\prime}
\end{aligned}
$$

which is the Kottler representation of the magnetic field. The surface integral in (9) is transformed into a line integral using [7, (10), (18), (22), (23)] with $\mathbf{E}^{i}$ replaced by $\mathbf{H}^{i}$. The result for $\mathbf{H}^{A}$ is then

$$
\begin{aligned}
\mathbf{H}^{A}(\mathbf{r})= & -\mathbf{H}^{i}(\mathbf{r}) \chi(\mathbf{r})+\int_{\Gamma} \hat{\mathbf{t}} \times \mathbf{H}^{i}\left(\mathbf{r}^{\prime}\right) G\left(\mathbf{r}, \mathbf{r}^{\prime}\right) d \Gamma^{\prime} \\
+ & \int_{\Gamma} \hat{\mathbf{t}} \cdot\left[\mathbf{V}\left(\mathbf{r}, \mathbf{r}^{\prime}\right) \mathbf{H}^{i}(\mathbf{r})+\overline{\mathbf{W}}_{H}\left(\mathbf{r}, \mathbf{r}^{\prime}\right)\right. \\
& \left.\quad+\frac{1}{j k Z} \mathbf{E}^{i}\left(\mathbf{r}^{\prime}\right) \nabla^{\prime} G\left(\mathbf{r}, \mathbf{r}^{\prime}\right)\right] d \Gamma^{\prime}
\end{aligned}
$$

The transformation from (9) to (10) is valid only if the Hertzian dipole is not located on the surface of the cone with vertex at $\mathbf{r}$ and generators extending from this point to the edge $\Gamma$, see [7, Fig. 2]. In (10) $\chi(\mathbf{r})=1$ if the Hertzian dipole is located inside the above-mentioned cone and zero otherwise. Furthermore, $\mathbf{V}\left(\mathbf{r}, \mathbf{r}^{\prime}\right)=\hat{\mathbf{p}} \times \hat{\mathbf{R}} /[4 \pi R(1-\hat{\mathbf{R}} \cdot \hat{\mathbf{p}})]$ with $\hat{\mathbf{p}}$ being a fixed arbitrary unit vector in the plane of the plate, and

$$
\overline{\mathbf{W}}_{H}\left(\mathbf{r}, \mathbf{r}^{\prime}\right)=\frac{1}{4 \pi} \hat{\mathbf{R}} \times \int_{0}^{1} \nabla^{\prime} \mathbf{H}^{i}(\tau \mathbf{R}) \exp (-j k \tau R) d \tau .
$$

The expression for the magnetic PO scattered field is finally obtained by inserting (10) into (3). This yields

$$
\begin{aligned}
& \mathbf{H}^{P O}\left(\mathbf{r}_{F}\right)=-\mathbf{H}^{i}\left(\mathbf{r}_{F}\right) \chi\left(\mathbf{r}_{F}\right)+\mathbf{H}^{i}\left(\mathbf{r}_{I}\right) \cdot(\overline{\mathbf{I}}-2 \hat{\mathbf{z}} \hat{\mathbf{z}}) \chi\left(\mathbf{r}_{I}\right) \\
&+\int_{\Gamma}\left\{\hat { \mathbf { t } } \cdot \left[\overline{\mathbf{W}}_{H}\left(\mathbf{r}_{F}, \mathbf{r}^{\prime}\right)-\overline{\mathbf{W}}_{H}\left(\mathbf{r}_{I}, \mathbf{r}^{\prime}\right) \cdot(\overline{\mathbf{I}}-2 \hat{\mathbf{z}} \hat{\mathbf{z}})\right.\right. \\
&\left.\quad+\mathbf{V}\left(\mathbf{r}_{F}, \mathbf{r}^{\prime}\right)\left(\mathbf{H}^{i}\left(\mathbf{r}_{F}\right)+\mathbf{H}^{i}\left(\mathbf{r}_{I}\right) \cdot(\overline{\mathbf{I}}-2 \hat{\mathbf{z}} \hat{\mathbf{z}})\right)\right] \\
& \\
&\left.\quad+2 \hat{\mathbf{z}} \hat{\mathbf{z}} \cdot\left(\hat{\mathbf{t}} \times \mathbf{H}^{i}\left(\mathbf{r}^{\prime}\right)\right) G\left(\mathbf{r}_{F}, \mathbf{r}^{\prime}\right)\right\} d \Gamma^{\prime} .
\end{aligned}
$$

If the expression for the dyadic $\overline{\mathbf{W}}_{H}$ in (11) can be evaluated analytically, (12) is the desired line integral representation of the magnetic

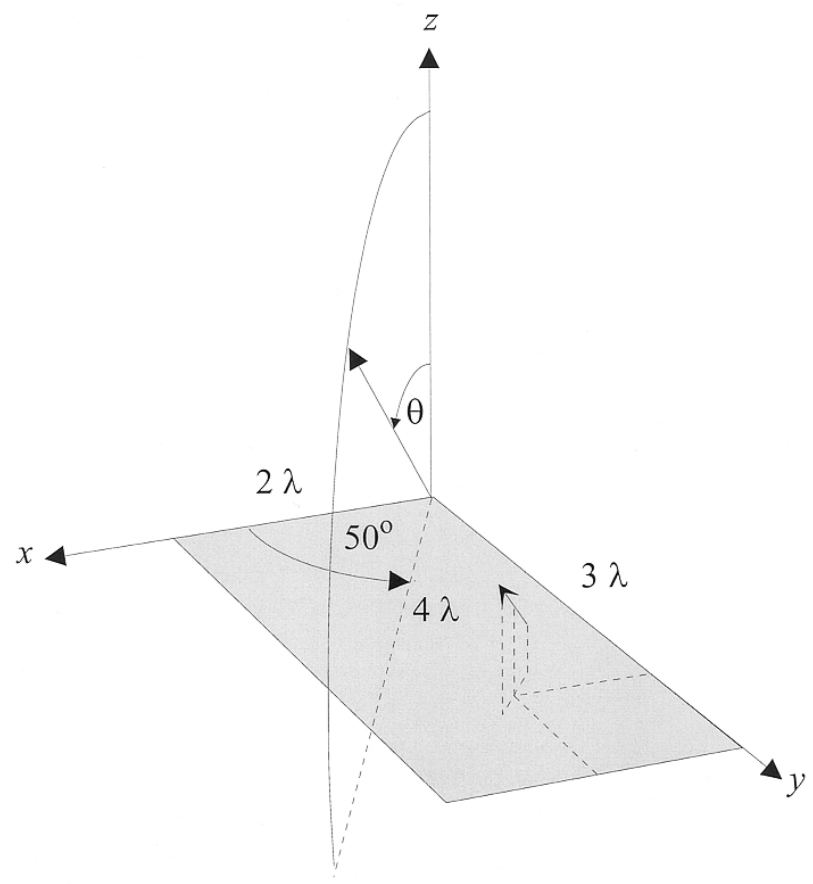

Fig. 2. A $2 \lambda$ by $3 \lambda$ plate illuminated by a Hertzian dipole located at $(1 \lambda, 2 \lambda$, $1 \lambda$ ). The observation points are at $r=4 \lambda$ in the $\phi=50^{\circ}$ plane.

PO scattered field. In the following, analytical expressions for (11) are derived for the two cases in which the illuminating source is an electric and a magnetic Hertzian dipole, respectively.

\section{A. Illumination by an Electric Hertzian Dipole}

The electric Hertzian dipole located at $\mathbf{r}=\mathbf{r}_{S}$ and with the dipole moment $\boldsymbol{\alpha}_{e}$ is described by the electric current density $\mathbf{J}_{e}(\mathbf{r})=\boldsymbol{\alpha}_{e} \delta\left(\mathbf{r}-\mathbf{r}_{S}\right)$, where $\delta$ is the Dirac delta function. The incident magnetic field is

$$
\mathbf{H}_{e}^{i}\left(\mathbf{r}^{\prime}\right)=G\left(\mathbf{r}^{\prime}, \mathbf{r}_{S}\right)\left(j k+\frac{1}{\rho}\right) \boldsymbol{\alpha}_{e} \times \hat{\boldsymbol{\rho}}
$$

where $\boldsymbol{\rho}=\rho \hat{\boldsymbol{\rho}}=\mathbf{r}^{\prime}-\mathbf{r}_{S}$. In the Appendix of this letter $\overline{\mathbf{W}}_{H}$ in (11) is calculated with the result

$$
\begin{aligned}
\overline{\mathbf{W}}_{H}\left(\mathbf{r}, \mathbf{r}^{\prime}\right)=\frac{1}{(4 \pi)^{2}}[ & K_{1}\left(\mathbf{A} \boldsymbol{\alpha}_{e} \times \mathbf{R}_{S}-\hat{\mathbf{R}} \cdot \mathbf{R}_{S} \mathbf{A B}\right) \\
& +K_{2}\left(\boldsymbol{\alpha}_{e} \hat{\mathbf{R}}-\overline{\mathbf{I}} \hat{\mathbf{R}} \cdot \boldsymbol{\alpha}_{e}+j k \mathbf{A B}\right) \\
& \left.+K_{3} \mathbf{A B}\right] .
\end{aligned}
$$

Herein, $\mathbf{A}=\hat{\mathbf{R}} \times \mathbf{R}_{S}, \mathbf{B}=\boldsymbol{\alpha}_{e} \times \hat{\mathbf{R}}, \mathbf{R}_{S}=R_{S} \hat{\mathbf{R}}_{S}=\mathbf{r}_{S}-\mathbf{r}$, and

$$
\begin{aligned}
K_{1}= & C_{1} \rho^{3}\left[j k C_{3}+C_{3}^{2}(2+\hat{\mathbf{R}} \cdot \hat{\boldsymbol{\rho}})\right] \\
& -C_{2} R_{S}^{2}\left(C_{4}\left(1+j k R_{S}\right)+C_{4}^{2}\right) \\
K_{2}= & C_{1} C_{3} \rho^{4}-C_{2} C_{4} R_{S}^{4} \\
K_{3}= & -C_{1} \rho^{2}(1+j k \rho)+C_{2} R_{S}^{3}\left(1+j k R_{S}\right) .
\end{aligned}
$$

The quantities $C_{1}, \ldots, C_{4}$ are identical to those given in [7, (35)-(38)]

$$
\begin{aligned}
& C_{1}=\left(R \rho^{5}\right)^{-1} \exp (-j k(R+\rho)) \\
& C_{2}=\left(R R_{S}^{6}\right)^{-1} \exp \left(-j k R_{S}\right) \\
& C_{3}=(\rho+\hat{\mathbf{R}} \cdot \rho)^{-1} \\
& C_{4}=\left(1-\hat{\mathbf{R}} \cdot \hat{\mathbf{R}}_{S}\right)^{-1} .
\end{aligned}
$$

Also, $K_{1}$ in (15) is identical to the one used in [7] whereas $K_{2}, K_{3}$ in (16), (17) are different from those defined in [7]. 


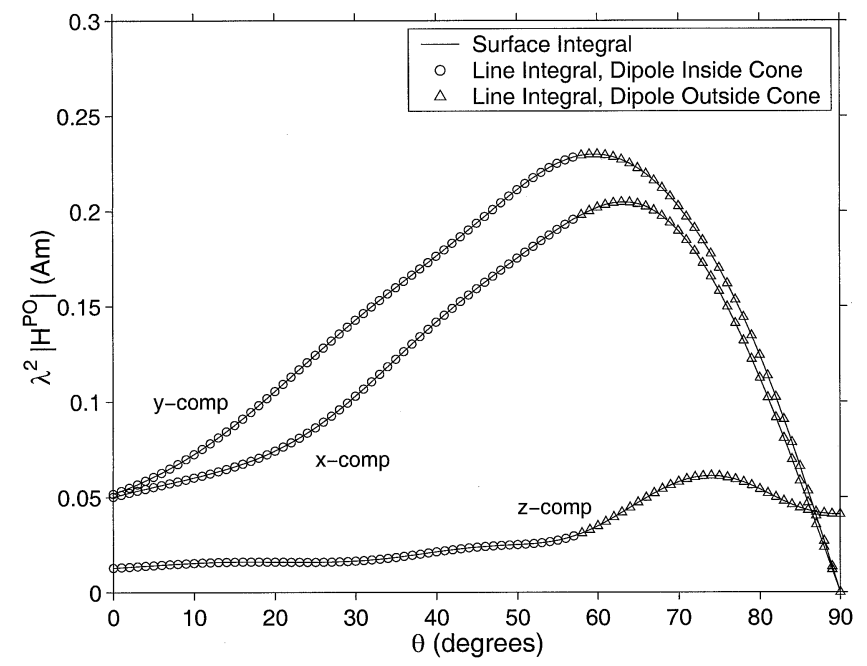

Fig. 3. Amplitudes of the three rectangular components of the magnetic PO scattered field for the configuration shown in Fig. 2. The source is an electric Hertzian dipole with dipole moment $\boldsymbol{\alpha}_{e}=(1,1,1) \mathrm{Am}$.

\section{B. Illumination by a Magnetic Hertzian Dipole}

The magnetic Hertzian dipole with the magnetic current density $\mathbf{J}_{m}(\mathbf{r})=\alpha_{m} \delta\left(\mathbf{r}-\mathbf{r}_{S}\right)$ radiates the magnetic field

$$
\begin{aligned}
\mathbf{H}_{m}^{i}\left(\mathbf{r}^{\prime}\right)= & \frac{-j G\left(\mathbf{r}^{\prime}, \mathbf{r}_{S}\right)}{k Z}\left[\left(-k^{2}+\frac{3 j k}{\rho}+\frac{3}{\rho^{2}}\right) \boldsymbol{\alpha}_{m} \cdot \hat{\boldsymbol{\rho}} \hat{\boldsymbol{\rho}}\right. \\
& \left.+\left(k^{2}-\frac{j k}{\rho}-\frac{1}{\rho^{2}}\right) \boldsymbol{\alpha}_{m}\right] .
\end{aligned}
$$

In [7, Appendix A], an analytical calculation of

$$
\overline{\mathbf{W}}=\frac{1}{4 \pi} \hat{\mathbf{R}} \times \int_{0}^{1} \nabla^{\prime} \mathbf{E}_{e}^{i}(\tau \mathbf{R}) \exp (-j k \tau R) d \tau
$$

is carried out where $\mathbf{E}_{e}^{i}$ is the electric field due to an electric Hertzian dipole. Since the magnetic field from a magnetic Hertzian dipole is related to the electric field from an electric Hertzian dipole through the principle of duality, it is seen that $\overline{\mathbf{W}}_{H}$ in (11) can be found from the result for $\overline{\mathbf{W}}$ in [7, (28)], using

$$
\overline{\mathbf{W}}_{H}=Z^{-1} \overline{\mathbf{W}} \text {. }
$$

In the expression for $\overline{\mathbf{W}}$ in [7, (28)], $\boldsymbol{\alpha}$ must be replaced by $Z^{-1} \boldsymbol{\alpha}_{m}$.

From application of the principle of duality to the incident field along with the result of [7, (24)], the closed-form expression for $\overline{\mathbf{W}}_{H}$ in (14) also makes it possible to derive a line integral representation of the electric PO field scattered by a planar plate when the illuminating source is a magnetic Hertzian dipole with dipole moment $\alpha_{m}$. This is done by setting $\overline{\mathbf{W}}=-Z \overline{\mathbf{W}}_{H}$ in [7, (24)], and by replacing $\boldsymbol{\alpha}_{e}$ by $Z^{-1} \boldsymbol{\alpha}_{m}$ in (14).

\section{NUMERICAL EXAMPLES}

Consider the rectangular planar PEC plate with dimensions $2 \lambda$ by $3 \lambda$ as shown in Fig. 2. The origin of the $x y z$ coordinate system is located at one corner of the plate and the illuminating Hertzian dipole is placed at $(1 \lambda, 2 \lambda, 1 \lambda)$. The observation points are located on the circular arc $r=4 \lambda$ in the $\phi=50^{\circ}$ plane with $\theta$ ranging from 0 to $90^{\circ}$. For this configuration, the Hertzian dipole is inside the cone with vertex at the observation point for $0 \leq \theta \leq 57.27^{\circ}$ and outside the cone for $57.27<\theta \leq 90^{\circ}$. In Fig. 3 the source is an electric Hertzian dipole with dipole moment $\boldsymbol{\alpha}_{e}=(1,1,1)$ Am and in Fig. 4 the source is a magnetic Hertzian dipole with dipole moment $\alpha_{m}=(376376376)$

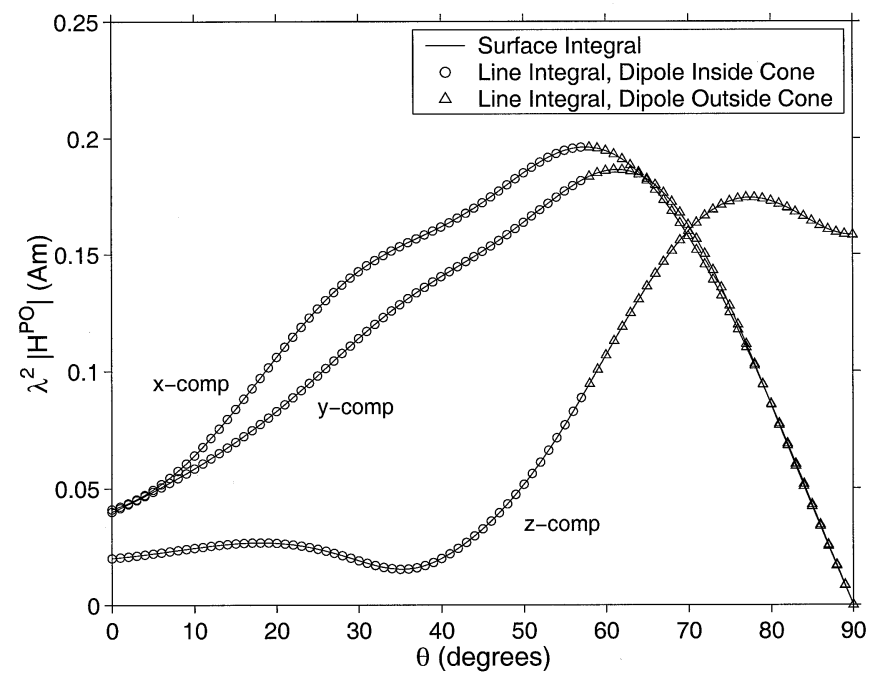

Fig. 4. Amplitudes of the three rectangular components of the magnetic PO scattered field for the configuration shown in Fig. 2. The source is a magnetic Hertzian dipole with dipole moment $\boldsymbol{\alpha}_{m}=(376376376) \mathrm{Vm}$.

$\mathrm{Vm}$. Both figures show the normalized amplitudes of the $x, y$ and $z$ components of the magnetic PO scattered field calculated both from the surface integral (1) and the line integral (12). As expected, exact agreement is observed. Similar agreement is found for the phases.

\section{CONCLUSION}

A line integral representation is derived of the magnetic PO field scattered by a planar PEC plate illuminated by either electric or magnetic Hertzian dipoles. The line integral representation is exact and it holds for almost any positions of source and observation points; the only exceptions are when the observation point is on the plate, and when the source point is located in the plane of the plate or on the surfaces of the cones with vertices at the observation and image points and generators extending from these points to the edge of the plate. These exceptions do not, however, constitute a severe limitation in practical applications. The results of this letter and that previously published in [7] make it possible to calculate as a line integral the electric as well as the magnetic PO scattered field from planar PEC plates illuminated by electric or magnetic Hertzian dipoles. Although the numerical example of the letter involves the scattering by a rectangular plate the PO line integral representation holds for arbitrarily shaped planar plates. The derivation of a PO line integral representation for the case in which the observation point is located in the far field has recently been completed, and the result published elsewhere [11]

\section{APPENDIX}

\section{Calculation of $\mathbf{W}_{H}$ FOR an Electric Dipole}

The gradient of the magnetic field (13) is

$$
\begin{aligned}
\nabla^{\prime} \mathbf{H}_{e}^{i}\left(\mathbf{r}^{\prime}\right)= & -G\left(\mathbf{r}^{\prime}, \mathbf{r}_{S}\right)\left[\left(\frac{j k}{\rho}+\frac{1}{\rho^{2}}\right) \overline{\mathbf{I}} \times \boldsymbol{\alpha}_{e}\right. \\
& \left.+\left(-k^{2}+\frac{3 j k}{\rho}+\frac{3}{\rho^{2}}\right) \hat{\rho} \boldsymbol{\alpha}_{e} \times \hat{\rho}\right] .
\end{aligned}
$$

Inserting this expression for $\nabla^{\prime} \mathbf{H}_{e}^{i}$ into (11) for $\overline{\mathbf{W}}_{H}$ we obtain

$$
\begin{aligned}
\overline{\mathbf{W}}_{H}\left(\mathbf{r}, \mathbf{r}^{\prime}\right)= & \frac{1}{(4 \pi)^{2}}\left[-\hat{\mathbf{R}} \times \mathbf{R}_{S} \alpha_{e} \times \mathbf{R} I_{1}\right. \\
& +\hat{\mathbf{R}} \times \mathbf{R}_{S} \alpha_{e} \times \mathbf{R}_{S} I_{2} \\
& \left.+\left(\alpha_{e} \hat{\mathbf{R}}-\overline{\mathbf{I}} \hat{\mathbf{R}} \cdot \alpha_{e}\right) I_{9}\right]
\end{aligned}
$$


where $\mathbf{R}_{S}=\mathbf{r}_{S}-\mathbf{r}$ and the integrals $I_{1}, I_{2}$, and $I_{9}$ are given by

$$
\begin{aligned}
& I_{1}=\int_{0}^{1} h\left(\frac{k^{2}}{f^{3}}-\frac{3 j k}{f^{4}}-\frac{3}{f^{5}}\right) \tau d \tau \\
& I_{2}=\int_{0}^{1} h\left(\frac{k^{2}}{f^{3}}-\frac{3 j k}{f^{4}}-\frac{3}{f^{5}}\right) d \tau \\
& I_{9}=\int_{0}^{1} h\left(\frac{-j k}{f^{2}}-\frac{1}{f^{3}}\right) d \tau
\end{aligned}
$$

with $f=f(\tau)=\left|\tau \mathbf{R}-\mathbf{R}_{S}\right|$ and $h=h(\tau)=\exp (-j k(\tau R+f))$. The results of the analytical evaluations of these integrals are given in [7, (51), (56), (61)]. Inserting these expressions for the integrals into (26), the final expression in (14) is obtained.

\section{REFERENCES}

[1] K. Miyamoto and E. Wolf, "Generalization of the Maggi-Rubinowicz theory of the boundary diffraction wave-part I," J. Opt. Soc. America, vol. 52, no. 6, pp. 615-625, June 1962.

[2] — , "Generalization of the Maggi-Rubinowicz theory of the boundary diffraction wave-Part II," J. Opt. Soc. America A, Opt. Image Sci., vol. 52, no. 6, pp. 626-637, June 1962.

[3] A. Rubinowicz, "The Miyamoto-Wolf diffraction wave," in Progress in Optics. Amsterdam, The Netherlands: North-Holland, 1965, vol. 4, pp. $199-240$.

[4] J. S. Asvestas, "Line integrals and physical optics. Part I. The transformation of the solid-angle surface integral to a line integral," J. Opt. Soc. America A, Opt. Image Sci., vol. 2, no. 6, pp. 891-895, June 1985.

[5] _ - "Line integrals and physical optics. Part II. The conversion of the Kirchhoff surface integral to a line integral," J. Opt. Soc. America A, Opt. Image Sci., vol. 2, no. 6, pp. 896-902, June 1985.

[6] — - "The physical optics fields of an aperture on a perfectly conducting screen in terms of line integrals," IEEE Trans. Antennas Propagat., vol. AP-34, pp. 1155-1159, Sept. 1986.

[7] P. Meincke Johansen and O. Breinbjerg, "An exact line integral representation of the physical optics scattered field: the case of a perfectly conducting polyhedral structure illuminated by electric Hertzian dipoles," IEEE Trans. Antennas Propagat., vol. 43, pp. 689-696, July 1995.

[8] G. Pelosi, G. Toso, and E. Martini, "PO near-field expression of a penetrable planar structure in terms of a line integral," IEEE Trans. Antennas Propagat., vol. 48, pp. 1274-1276, Aug. 2000.

[9] D. S. Jones, The Theory of Electromagnetism. New York: Pergamon, 1964, p. 635.

[10] G. Chambers, A Course in Vector Analysis. London, U.K.: Chapman \& Hall, 1969.

[11] S. Arslanagić, P. Meincke, E. Jørgensen, and O. Breinbjerg, "An exact line integral representation of the physical optics far field from plane PEC scatterers illuminated by Hertzian dipoles," J. Electromagnetic Waves Applicat., vol. 17, pp. 51-69, Jan. 2003.

\section{Polynomial Approximations to Bessel Functions}

\author{
R. P. Millane and J. L. Eads
}

\begin{abstract}
A polynomial approximation to Bessel functions that arises from an electromagnetic scattering problem is examined. The approximation is extended to Bessel functions of any integer order, and the relationship to the Taylor series is derived. Numerical calculations show that the polynomial approximation and the Taylor series truncated to the same order have similar accuracies.
\end{abstract}

Index Terms-Approximation, Bessel functions, polynomial.

\section{INTRODUCTION}

Bessel functions appear in numerous physical problems, and play an important role in many electromagnetic scattering problems. There is no closed form expression for Bessel functions so that approximations suitable for numerical evaluation are necessary in applications. Gross [1] has derived interesting polynomial approximations to the zerothand first-order Bessel functions of the first kind for small arguments, that arise from an integral that occurs in an electromagnetic scattering problem. We study here in detail properties of these approximations. First we extend the analysis in [1] to derive corresponding polynomial approximations for Bessel functions of any integer order. Second we show that as the degree of the polynomial approximation increases, it converges to the Taylor series expansion. Third we compare the accuracy of the polynomial approximations to that of the truncated Taylor series of the same order.

\section{BACKGROUND}

Gross [1] begins by considering the integral

$$
f_{2 n}(k)=\frac{2}{\pi} \int_{0}^{\delta} \frac{\cos x \cos (2 n x)}{\sqrt{k^{2}-\sin ^{2} x}} d x, \quad 0 \leq k \leq 1
$$

where

$$
k=\sin \delta, \quad 0 \leq \delta \leq \frac{\pi}{2}
$$

that occurs in the expression for the current density on a conducting strip grating illuminated by a plane electromagnetic wave [2]. The integral is evaluated as [1]

$$
f_{2 n}(k)=\sum_{m=0}^{n} b_{m} k^{2 n-2 m}
$$

where

$$
b_{m}=\frac{n(-1)^{m+n}(2 n-m-1) ! 2^{2 n-2 m}}{m !((2 n-2 m) ! !)^{2}}
$$

and $(2 n) ! !=2 \cdot 4 \cdot 6 \cdots 2 n$. Making the substitution $x=w \delta$ in (1) gives

$$
f_{2 n}(\sin \delta)=\frac{2}{\pi} \int_{0}^{1} \frac{\cos (\delta w) \cos (2 n \delta w)}{\sqrt{\sin ^{2} \delta-\sin ^{2}(\delta w)}} \delta d w
$$

Manuscript received January 18, 2001; revised August 28, 2001.

R. P. Millane is with the Department of Electrical and Computer Engineering, University of Canterbury, Christchurch, New Zealand (e-mail: rick@elec.canterbury.ac.nz).

J. L. Eads is with the Computational Science and Engineering Program, Purdue University, West Lafayette, IN 47907 USA.

Digital Object Identifier 10.1109/TAP.2003.812234 